\title{
Alteraciones bioquímicas como marcadores predictores de gravedad en pacientes con fiebre por dengue
}

\author{
Luis Ángel Villar-Centeno ${ }^{1,4}$, Anyela Lozano-Parra ${ }^{1,4}$, Doris Salgado-García $a^{2,4}$, Óscar F. Herrán ${ }^{3,4}$ \\ 1 Grupo de Epidemiología Clínica, Centro de Investigaciones Epidemiológicas, Universidad Industrial de \\ Santander, Bucaramanga, Colombia \\ 2 Grupo de Parasitología y Medicina Tropical, Universidad Surcolombiana, Neiva, Colombia \\ 3 Observatorio Epidemiológico de Enfermedades Cardiovasculares, Centro de Investigaciones Epidemiológicas, \\ Universidad Industrial de Santander, Bucaramanga, Colombia. \\ ${ }^{4}$ Red AEDES: Abordando Áreas Endémicas del Dengue para el Estudio de su Severidad (sic.), Colombia
}

Introducción. El dengue es la infección transmitida por mosquitos más importante en el mundo. Existe información de que las alteraciones bioquímicas pueden utilizarse como herramientas predictoras de gravedad del dengue.

Objetivo. Evaluar las alteraciones bioquímicas como posibles marcadores predictores de gravedad del dengue.

Materiales y métodos. Se llevó a cabo un estudio de casos y controles anidado en una cohorte. Se seleccionaron al azar 125 casos con dengue grave y 120 controles con dengue no grave para evaluar los niveles séricos de lactato-deshidrogenasa (LDH), creatina cinasa (CK), proteína C reactiva (PCR) y albúmina, en sueros obtenidos en las primeras horas de la enfermedad. Para evaluar el valor diagnóstico de cada biomarcador, se establecieron puntos de corte con una sensibilidad del $90 \%$ en la detección de casos graves.

Resultados. Se observó una asociación entre los niveles de PCR por debajo de 9,8 mg/l (OR=0,04; $\left.I C_{95 \%}=0,02-0,08 ; p=0,000\right)$, de LDH inferiores a $400 \mathrm{U} / \mathrm{L}\left(\mathrm{OR}=0,49 ; \mathrm{IC}_{95 \%}=0,24-1,02 ; p=0,053\right)$ y de albúmina menor de $4 \mathrm{mg} / \mathrm{dl}\left(\mathrm{OR}=3,46 ; \mathrm{IC}_{95 \%}=1,96-6,12 ; \mathrm{p}=0,000\right)$, con la gravedad del dengue. En contraste, los niveles de la CK no mostraron asociación con la gravedad de la enfermedad.

Conclusiones. Los hallazgos de nuestro estudio sugieren una asociación de los niveles de PCR, LDH y albúmina con la gravedad del dengue. Estas pruebas bioquímicas podrían ser utilizadas como herramientas predictoras del curso clínico de la infección.

Palabras clave: dengue, índice de gravedad de la enfermedad, marcadores biológicos, predicción, bioquímica, humanos.

doi: http://dx.doi.org/10.7705/biomedica.v33i0.732

Biochemical alterations as prediction markers for the severity of illness in dengue fever patients

Introduction: Dengue is the most important mosquito-borne infection in the world. There is evidence supporting the use of biochemical alterations as prediction tools for severity of illness in dengue.

Objective: To evaluate biochemical alterations as potential prediction markers for severity in dengue. Materials and methods: This was a case-control study nested in a cohort. We randomly selected 125 severe dengue cases and 120 controls with non-severe dengue for measuring LDH, CK, CRP and albumin serum levels using acute phase sera. To evaluate the predictive value for each biomarker, we established cut-off points with $90 \%$ sensitivity in detecting severe cases.

Results: There was association among the CRP levels $<9.8 \mathrm{mg} / \mathrm{L}(\mathrm{OR}=0.04 ; 95 \% \mathrm{Cl}=0.02-0.08$; $p=0.000),<400 \mathrm{U} / \mathrm{L}$ LDH levels $(\mathrm{OR}=0.49 ; 95 \% \mathrm{Cl}=0.24-1.02 ; \mathrm{p}=0.053)$ and $<4 \mathrm{mg} / \mathrm{dl}$ albumin levels $(\mathrm{OR}=3.46 ; 95 \% \mathrm{Cl}=1.96-6.12 ; \mathrm{p}=0.000)$ with the severity of dengue. In contrast, the CK levels showed no association with the severity of the disease.

Conclusions: Our findings suggest an association of CRP, LDH and albumin levels with the severity of dengue. These biochemical tests could be used as predictive tools in the clinical course of the infection.

\section{Contribución de los autores:}

Luis Villar-Centeno aportó el diseño del estudio, el análisis y la interpretación de los datos, la redacción y la revisión crítica del manuscrito.

Anyela Lozano-Parra contribuyó en la recolección y la obtención de los resultados, la interpretación de los datos y la redacción del manuscrito.

Doris Salgado-García brindó soporte en la redacción del manuscrito.

Óscar F. Herrán contribuyó en el análisis y la interpretación de los datos y la redacción del manuscrito. 
Keywords: dengue, severity of illness index, biological markers, forecasting, biochemistry, humans.

doi: http://dx.doi.org/10.7705/biomedica.v33i0.732

El dengue actualmente es la enfermedad transmitida por mosquitos de mayor impacto mundial (1). Durante la última década, su incidencia se ha incrementado debido a la adaptación de su vector Aedes aegypti a la creciente concentración de la población en áreas urbanas, el gran movimiento humano y la expansión de la infección hacia nuevas regiones del trópico y áreas subtropicales del mundo $(2,3)$.

Su expresión clínica es de amplio espectro; varía desde cuadros febriles de resolución espontánea que se acompañan de cefalea, mialgias, artralgias y dolor retroocular, hasta cuadros graves (aproximadamente, el $15 \%$ de los casos clínicos) cuya letalidad oscila entre 2 y $4 \%$ (1) y podría reducirse mediante una identificación temprana de los enfermos con mayor riesgo de complicaciones (4).

Aún no existe una alternativa terapéutica específica para el tratamiento del dengue. De acuerdo con las Guías de Manejo de la Organización Mundial de la Salud (OMS), el manejo médico debe concentrarse en la administración oportuna y suficiente de líquidos (por vía oral o parenteral, según las condiciones del enfermo), para evitar complicaciones mediante el tratamiento sintomático y un seguimiento clínico estricto (3).

Puesto que la evolución del dengue en los primeros días de la enfermedad no es predecible, se requieren herramientas que orienten tempranamente al personal de salud en la identificación de los enfermos de mayor riesgo de evolucionar hacia formas graves, con el fin de priorizar su atención y brindar un cuidado específico y oportuno. Unas de estas herramientas podrían ser algunos marcadores biológicos ya disponibles, de alto rendimiento, complejidad técnica alcanzable y costos razonables (5).

Existen datos que sugiere una asociación temprana de los niveles elevados de la lactatodeshidrogenasa (LDH) (6-9), la creatina-cinasa (CK) (9-12) y la proteína C reactiva (PCR) (12-14),

\footnotetext{
Correspondencia:

Óscar Fernando Herrán, Carrera 32 № 29-31, oficina 304, Centro de Investigaciones Epidemiológicas, Facultad de Salud, Universidad Industrial de Santander, La Aurora, Bucaramanga. Teléfono: (577) 634 4000, extensión 3205; Fax: (577) 6345781 herran@uis.edu.co

Recibido: 04/05/12; aceptado:04/10/12
}

con las complicaciones por dengue. Otro tanto sucede con los niveles bajos de albúmina $(9,10)$. Esta observación requiere de una cuidadosa valoración en el contexto de un estudio de cohorte. En este estudio se examina la potencial asociación entre las alteraciones tempranas de LDH, CK, PCR y albúmina, y la gravedad de la enfermedad.

\section{Materiales y métodos}

\section{Tipo de estudio}

Se llevó a cabo un estudio de casos y controles anidado en una cohorte.

\section{Descripción de la población}

Se trata de pacientes con síndrome febril agudo y diagnóstico confirmado de dengue, residentes en alguna de las regiones de alta circulación del virus y frecuencia de enfermedad grave en Colombia.

En el presente estudio se incluyeron pacientes de un año o más de edad con síndrome febril agudo sin foco aparente durante las primeras horas de la enfermedad, después del examen físico, de 11 centros de captación (cuadro 1).

De esta población se excluyeron aquellos con antecedentes de diabetes, alteraciones hematológicas, cáncer, $\mathrm{VIH} /$ sida, enfermedad cardiaca $\mathrm{O}$ consumo de corticoides en los últimos ocho días. Tampoco ingresaron al estudio quienes al momento inicial presentaron manifestaciones clínicas de complicaciones de dengue grave, como sangrado mayor o hipotensión (signos de gravedad).

La infección por dengue se confirmó mediante seroconversión (viraje de negativo a positivo o cuadruplicación de los títulos de anticuerpos IgM específicos para dengue en muestras de fase aguda y de fase de convalecencia), aislamiento viral, detección de proteína no estructural NS1 por ELISA (Enzyme-Linked ImmunoSorbent Assay), inmunocromatografía, o reacción en cadena de la polimerasa de la transcriptasa inversa (RT-PCR) cualitativa.

\section{Captación y seguimiento}

Después de la obtención del consentimiento informado, se practicó un examen físico inicial y se obtuvo una muestra de sangre para un cuadro hemático con recuento de plaquetas, pruebas diagnósticas del dengue y medición de marcadores bioquímicos. 
Cuadro 1. Centros de captación de los participantes del estudio

\begin{tabular}{ll}
\hline Ciudad & Centro \\
\hline Barranquilla & $\begin{array}{l}\text { Consulta Externa, Centro de Salud, } \\
\text { Hospital del Norte } \\
\text { Hospital Pediátrico de Barranquilla }\end{array}$ \\
$\begin{array}{l}\text { Bucaramanga y } \\
\text { área metropolitana }\end{array}$ & $\begin{array}{l}\text { Hospital San Juan de Dios de Floridablanca } \\
\text { Clíndación Oftalmológica Carlos Ardila }\end{array}$ \\
& $\begin{array}{l}\text { Lulle, Hospital Local de Piedecuesta } \\
\text { Hospital Local del Norte }\end{array}$ \\
Palmira & Hospital Universitario de Santander. \\
Cali & Hospital Raúl Orejuela Bueno \\
Neiva & Hospital Universitario del Valle EGA \\
\hline
\end{tabular}

Los pacientes fueron seguidos diariamente hasta la resolución de sus síntomas o, al menos, hasta el sexto día de enfermedad; cada día se evaluó y registró la aparición de complicaciones como hipotensión, sangrado, trombocitopenia, signos de extravasación de plasma, compromiso visceral, falla multisistémica o muerte.

Entre el día 7 y el 15 del inicio de los síntomas, se obtuvo una muestra de suero para practicar pruebas de IgM de fase de convalecencia. Los sueros obtenidos durante el estudio se almacenaron a $-70{ }^{\circ} \mathrm{C}$ hasta la realización de las pruebas de diagnóstico y bioquímicas.

El protocolo de este trabajo fue aprobado por el Comité de Ética de la Facultad de Salud de la Universidad Industrial de Santander.

\section{Definición de casos y controles}

Se consideraron como "casos" los pacientes que durante el seguimiento desarrollaron formas graves de la enfermedad, que presentaron, junto con un recuento de plaquetas inferior a $100.000 / \mathrm{mm}^{3}$, alguna manifestación hemorrágica (como hematemesis, melenas, rectorragia, menometrorragia, hematuria microscópica, equimosis, hematomas, hemartrosis, epistaxis o gingivorragia grave), extravasación plasmática (documentada por derrames serosos, viraje del hematocrito mayor o igual al $20 \%$ ) y falla hemodinámica (manifestada por presión arterial media inferior a $70 \mathrm{~mm} \mathrm{Hg} 0$ presión de pulso por debajo de $20 \mathrm{~mm} \mathrm{Hg}$ ).

Se consideraron como controles de dengue no grave, los pacientes con infección por virus del dengue que no desarrollaron complicaciones durante su enfermedad.

\section{Selección de casos y controles}

Para la selección de los sujetos del estudio, los pacientes fueron clasificados como casos o controles de acuerdo con su evolución clínica. Se creó un listado de 1.372 códigos pareados por sexo y edad, del cual se seleccionaron al azar 125 casos y 120 controles para la evaluación de las alteraciones bioquímicas durante las primeras 96 horas de la enfermedad.

\section{Medición de marcadores bioquímicos}

Las pruebas bioquímicas se practicaron en muestras de la fase aguda de la enfermedad. Se midieron los niveles de CK, LDH (pruebas LiquiUV modificadas) y albúmina (pruebas colorimétricas), utilizando reactivos de la casa comercial Randox ${ }^{\circledR}$, en el equipo Vitalab Selectra Junior ${ }^{\circledR}$, Merck. La PCR cuantitativa se practicó mediante nefelometría, con reactivos de la casa comercial Byosistems en el equipo Thermo Scientific Konelab $30{ }^{\circledR}$.

\section{Análisis de datos}

Los datos se almacenaron en una base electrónica en Excel MS Office 2007. Se describieron las características sociodemográficas y clínicas de la población de estudio, utilizando las proporciones expresadas en porcentajes. Los resultados de las pruebas bioquímicas se expresaron como medidas de tendencia central y dispersión (media con la desviación estándar en la albúmina y, mediana con los valores mínimo y máximo, para LDH, CK y PCR).

Inicialmente, se compararon los niveles de los marcadores estudiados en los casos y controles, buscando diferencias entre los grupos. Para esto, se hizo un análisis bivariado mediante la prueba $t$ de Student, cuando la distribución del biomarcador fue normal; de lo contrario, se utilizó la prueba $U$ de Mann-Whitney.

Posteriormente, mediante curvas ROC se determinaron los puntos de corte para cada parámetro bioquímico (excepto la albúmina) con una sensibilidad de $90 \%$ para detectar casos de dengue grave. En la albúmina se empleó el límite inferior aceptado como valor normal $(4 \mathrm{mg} /$ dl). Para evaluar la asociación de cada marcador sérico en los casos graves se estimó el odds ratio (OR) de los valores inferiores al punto de corte, con su respectivo intervalo de confianza del $95 \%$ $\left(\mathrm{IC}_{95 \%}\right)$. Cuando el valor de $\mathrm{p}$ era menor de 0,05 , la asociación se consideraba estadísticamente significativa. En el procesamiento de los datos se 
empleó el programa estadístico Stata ${ }^{\mathrm{TM}}$, versión 10.1 (Stata Corp.).

\section{Resultados}

Se incluyeron 125 casos de dengue grave y 120 controles de dengue no grave, captados durante el periodo 2003-2010.

El rango de edad para los sujetos incluidos fue de 1 a 73 años. La población infantil entre 5 y 14 años, predominó en el estudio (64\%). En los grupos evaluados, no se encontraron diferencias estadísticamente significativas según edad, sexo ni tiempo de evolución de la enfermedad (cuadro 2).

Se encontraron diferencias estadísticamente significativas en los niveles de los marcadores bioquímicos LDH, PCR y albúmina $(p<0,05)$, pero no en los de la CK. Los niveles de LDH y PCR fueron más altos ( $p=0,004$ y $p=0,000$, respectivamente) y de albúmina más bajos $(p=0,000)$, en los casos que en los controles (cuadro 3).

Los puntos de corte fijados mediante las curvas ROC para las pruebas bioquímicas, fueron los siguientes: LDH, $400 \mathrm{U} / \mathrm{L}, \mathrm{CK}, 48 \mathrm{mg} / \mathrm{dl}$, y PCR, 9,8 $\mathrm{mg} / \mathrm{L}$. En la albúmina se empleó el límite inferior normal de $4 \mathrm{mg} / \mathrm{dl}$ (cuadro 4).

Al establecer asociación con la gravedad, se encontró que, al inicio de la enfermedad, tener niveles de PCR por debajo de 9,8 mg/dl (OR=0,04; $\left.\mathrm{IC}_{95 \%}=0,02-0,08 ; p=0,000\right)$ y $\mathrm{LDH}$ menor de 400 $\mathrm{U} / \mathrm{L}\left(\mathrm{OR}=0,49 ; \quad \mathrm{IC}_{95 \%}=0,24-1,02 ; \mathrm{p}=0,053\right)$, podía disminuir el riesgo de evolucionar a la forma grave del dengue; en contraste, tener niveles de albúmina inferiores a $4 \mathrm{mg} / \mathrm{dl}$ podía incrementar 3,46 veces el riesgo de presentar un cuadro clínico complicado $\left(\mathrm{OR}=3,46 ; \mathrm{IC}_{95 \%}=1,96-6,12 ; \mathrm{p}=0,000\right)$ (cuadro 4). En el caso de la CK, no se encontraron diferencias en el desarrollo de dengue grave y dengue no grave $(p=0,557)$.

\section{Discusión}

Este trabajo aborda la evaluación de los niveles de LDH, CK, PCR y albúmina, como marcadores tempranos de gravedad en la infección por dengue.

Al evaluar la asociación entre los niveles de PCR con la gravedad se encontró que, en los primeros días de enfermedad, tener niveles inferiores a 9,8 $\mathrm{mg} / \mathrm{L}$ podía reducir el riesgo de sufrir posteriores

Cuadro 2. Descripción de las características demográficas y clínicas basales de los pacientes con dengue y alteraciones hemodinámicas durante el seguimiento

\begin{tabular}{|c|c|c|c|c|c|c|c|}
\hline \multirow{2}{*}{$\frac{\text { Variable }}{\text { Sexo, n (\%) }}$} & \multicolumn{2}{|c|}{ Casos $(n=125)$} & \multicolumn{2}{|c|}{ Controles $(n=120)$} & \multicolumn{2}{|r|}{ Total } & \multirow[t]{2}{*}{ p } \\
\hline & & & & & & & \\
\hline Femenino & 59 & $(47,2)$ & 56 & $(46,7)$ & 115 & $(46,9)$ & 0,933 \\
\hline Masculino & 66 & $(52,8)$ & 64 & $(53,3)$ & 130 & $(53,1)$ & \\
\hline \multicolumn{8}{|l|}{ Edad (años), n (\%) } \\
\hline $0-4$ & 19 & $(15,2)$ & 13 & $(10,8)$ & 32 & $(13,1)$ & 0,594 \\
\hline $5-14$ & 79 & $(63,2)$ & 79 & $(65,8)$ & 158 & $(64,5)$ & \\
\hline 15 y más & 27 & $(21,6)$ & 28 & $(23,3)$ & 55 & $(22,4)$ & \\
\hline \multicolumn{8}{|l|}{ Horas de enfermedad, $n(\%)$} \\
\hline$<96$ & 86 & $(68,8)$ & 90 & $(75,0)$ & 176 & $(71,8)$ & 0,281 \\
\hline$>96$ & 39 & $(31,2)$ & 30 & $(25,0)$ & 69 & $(28,2)$ & \\
\hline \multicolumn{8}{|l|}{ Manifestaciones, n (\%) } \\
\hline Fiebre & 29 & $(23,4)$ & 18 & $(15,1)$ & 47 & $(19,3)$ & 0,103 \\
\hline Cefalea & 85 & $(78,7)$ & 88 & $(80,0)$ & 173 & $(79,4)$ & 0,813 \\
\hline Dolor retroocular & 46 & $(43,0)$ & 50 & $(45,5)$ & 96 & $(44,2)$ & 0,785 \\
\hline Mialgias & 79 & $(73,8)$ & 79 & $(71,8)$ & 158 & $(72,8)$ & 0,762 \\
\hline Vómito & 61 & $(49,6)$ & 61 & $(57,0)$ & 122 & $(53,0)$ & 0,261 \\
\hline Diarrea & 45 & $36,6)$ & 37 & $(31,1)$ & 82 & $(33,9)$ & 0,367 \\
\hline Prueba de torniquete basal & 27 & $(21,8)$ & 5 & $(4,3)$ & 32 & $(13,3)$ & 0,000 \\
\hline \multicolumn{8}{|l|}{ Complicaciones* ${ }^{\star} \mathrm{n}(\%)$} \\
\hline \multicolumn{8}{|l|}{ Deshidratación } \\
\hline No & 67 & $(54,0)$ & 92 & $(77,3)$ & 159 & $(65,4)$ & 0,000 \\
\hline Sí & 57 & $(46.0)$ & 27 & $(22.7)$ & 84 & $(34.6)$ & \\
\hline Hipotensión ortostática & 2 & $(1,6)$ & 4 & $(3,4)$ & 6 & $(2,5)$ & 0,439 \\
\hline Presión arterial media $\leq 70 \mathrm{~mm}$ de $\mathrm{Hg}$ & 124 & $(100,0)$ & 2 & $(2,1)$ & 126 & $(57,8)$ & 0,000 \\
\hline Presión de pulso $\leq 20 \mathrm{~mm}$ de $\mathrm{Hg}$ & 35 & $(28,7)$ & 1 & $(0,9)$ & 36 & $(15,3)$ & 0,000 \\
\hline
\end{tabular}

*Documentados durante el seguimiento 
Cuadro 3. Resultados de las pruebas bioquímicas realizadas en la fase aguda de la enfermedad por dengue

\begin{tabular}{|c|c|c|c|c|c|c|c|}
\hline Variable & $\begin{array}{c}\text { Dengue } \\
\text { grave }\end{array}$ & $\mathbf{n}$ & Mediana & ( Q1 - Q3) & Mínimo & Máximo & Valor $p$ \\
\hline LDH & $\begin{array}{c}\text { Sí } \\
\text { No } \\
\text { Total }\end{array}$ & $\begin{array}{l}125 \\
120 \\
245\end{array}$ & $\begin{array}{l}672,0 \\
614,5 \\
631,0\end{array}$ & $\begin{array}{c}(524-1020) \\
(436,5-757,5) \\
(496-833)\end{array}$ & $\begin{array}{l}292,0 \\
179,0 \\
179,0\end{array}$ & $\begin{array}{l}9760,0 \\
2058,0 \\
9760,0\end{array}$ & 0,004 \\
\hline CK & $\begin{array}{c}\text { Sí } \\
\text { No } \\
\text { Total }\end{array}$ & $\begin{array}{l}125 \\
120 \\
245\end{array}$ & $\begin{array}{l}136,0 \\
167,5 \\
144,0\end{array}$ & $\begin{array}{c}(80-387) \\
(77-910,5) \\
(79-593)\end{array}$ & $\begin{array}{r}10,0 \\
8,0 \\
8,0\end{array}$ & $\begin{array}{l}4530,0 \\
4040,0 \\
4530,0\end{array}$ & 0,275 \\
\hline $\begin{array}{l}\text { Albúmina* } \\
\text { Total }\end{array}$ & $\begin{array}{l}\text { Sí } \\
\text { No } \\
245\end{array}$ & $\begin{array}{l}125 \\
120 \\
4,3\end{array}$ & $\begin{array}{l}4,0 \\
4,6 \\
0,7\end{array}$ & $\begin{array}{l}0,6 \\
0,8 \\
1,9\end{array}$ & $\begin{array}{l}2,5 \\
1,9 \\
7,4\end{array}$ & $\begin{array}{l}5,4 \\
7,4\end{array}$ & 0,000 \\
\hline PCR & $\begin{array}{l}\text { Sí } \\
\text { No } \\
\text { Total }\end{array}$ & $\begin{array}{l}125 \\
120 \\
245\end{array}$ & $\begin{array}{r}12,2 \\
4,2 \\
10,6\end{array}$ & $\begin{array}{c}(10,6-15,9) \\
(1,2-10,3) \\
(3,6-14,8)\end{array}$ & $\begin{array}{l}0,0 \\
0,0 \\
0,0\end{array}$ & $\begin{array}{l}220,4 \\
122,0 \\
220,4\end{array}$ & 0,000 \\
\hline
\end{tabular}

LDH: lactato-deshidrogenasa; CK: creatina-cinasa; PCR: proteína C reactiva

* Media y desviación estándar (prueba t).

Cuadro 4. Concentración sérica de LDH, CK, PCR y albúmina y su asociación con el resultado clínico

\begin{tabular}{|c|c|c|c|c|c|c|}
\hline Bioquímico & & $\begin{array}{c}\text { Casos } \\
(n=125)\end{array}$ & $\begin{array}{c}\text { Controles } \\
(n=120)\end{array}$ & Total & OR IC I5\% $_{1}$ & p \\
\hline $\mathrm{LDH}$ & $\begin{array}{l}>400 \mathrm{U} / \mathrm{L} \\
\leq 400 \mathrm{U} / \mathrm{L}\end{array}$ & $\begin{aligned} 112 & (89,6) \\
13 & (10,4)\end{aligned}$ & $\begin{array}{ll}97 & (80,8) \\
23 & (19,2)\end{array}$ & $\begin{aligned} 209 & (85,3) \\
36 & (14,7)\end{aligned}$ & $0,49(0,24-1,02)$ & 0,053 \\
\hline CK & $\begin{array}{l}>48 \mathrm{U} / \mathrm{L} \\
\leq 48 \mathrm{U} / \mathrm{L}\end{array}$ & $\begin{array}{rr}113 & (90,4) \\
12 & (9,6)\end{array}$ & $\begin{array}{rr}111 & (92,5) \\
9 & (7,5)\end{array}$ & $\begin{array}{rr}224 & (91,4) \\
21 & (8,6)\end{array}$ & $1,31(0,53-3,23)$ & 0,557 \\
\hline PCR & $\begin{array}{l}>9,8 \mathrm{mg} / \mathrm{L} \\
\leq 9,8 \mathrm{mg} / \mathrm{L}\end{array}$ & $\begin{aligned} 112 & (89,6) \\
13 & (10,4)\end{aligned}$ & $\begin{array}{ll}31 & (25,8) \\
89 & (74,2)\end{array}$ & $\begin{array}{ll}143 & (58,4) \\
102 & (41,6)\end{array}$ & $0,04(0,02-0,08)$ & 0,000 \\
\hline Albúmina & $\begin{array}{l}>4 \mathrm{mg} / \mathrm{dl} \\
\leq 4 \mathrm{mg} / \mathrm{dl}\end{array}$ & $\begin{array}{ll}67 & (53,6) \\
58 & (46,4)\end{array}$ & $\begin{array}{ll}96 & (80,0) \\
24 & (20,0)\end{array}$ & $\begin{aligned} 163 & (66,5) \\
82 & (33,5)\end{aligned}$ & $3,46(1,96-6,12)$ & 0,000 \\
\hline
\end{tabular}

LDH: lactato-deshidrogenasa; CK: creatina-cinasa; PCR: proteína $\mathrm{C}$ reactiva

complicaciones. Debido a que la PCR hace parte de las proteínas sintetizadas durante la fase aguda de un proceso infeccioso o inflamatorio, consideramos que el aumento en los niveles de PCR es influenciado por la infección en curso. La síntesis de proteínas de la fase aguda en el hígado es estimulada principalmente por la interleucina 6 (IL-6) (13,15). Esta teoría se fundamenta en el hecho de que, durante las primeras horas de la enfermedad, se desencadena la liberación de una cascada de citocinas (una de las más abundante es la IL-6) como respuesta al proceso infeccioso, fenómeno que juega un papel importante en su patogenia $(1-3,16)$.

En los estudios sobre dengue en que se evaluó la PCR, se emplearon técnicas semicuantitativas; en este estudio, para obtener resultados que reflejen el estado real de esta proteína, la cuantificación se hizo mediante pruebas ultrasensibles. En enfermedades con otras causas, como la endocarditis infecciosa y el sarampión, se ha propuesto esta proteína como marcador de gravedad $(17,18)$. Olaison, et al., encontraron que los niveles de PCR altos se asocian significativamente con episodios complicados de endocarditis infecciosa y propusieron utilizar mediciones seriadas para detectar oportunamente las complicaciones que se presenten durante el curso clínico de la enfermedad (17). Asimismo, Roine, et al., plantearon emplear el aumento de la PCR cuantitativa como un signo de alarma para complicaciones en el sarampión (18).

La LDH también podría ser útil en la predicción del dengue grave. En la literatura científica hay reportes de niveles elevados de este marcador asociados con síntomas característicos de gravedad (6-9). Durante la infección por el virus del dengue, se genera una afección del miocito de los músculos estriados, lo que podría desencadenar la liberación de LDH $(9,19)$. Otra manifestación frecuente en el dengue es el compromiso hepático, el cual también podría estar asociado con el incremento en los niveles de LDH $(20,21)$. Ejemplo 
de lo anterior es lo descrito por Agrawal, et al., en un modelo animal, donde obervaron que, al inocular el virus del dengue, los niveles de LDH en el músculo esquelético se incrementaban con el paso de los días (19). Además, en una cohorte de 199 pacientes con dengue se encontró que tener, al inicio de la enfermedad, LDH mayor de $570 \mathrm{U} / \mathrm{L}$ incrementaba el riesgo de evolucionar a la forma complicada de la enfermedad $(\mathrm{OR}=3,19$; $\left.\mathrm{Cl}_{95 \%}=1,01-10,12 ; p<0,05\right)(9)$. Nuestros resultados corroboran esta asociación, con un punto de corte más bajo de LDH (400 U/L).

Con la albúmina se confirman los resultados de anteriores estudios en los que se afirma que tener niveles por debajo de $4 \mathrm{mg} / \mathrm{dl}$ incrementa el riesgo de dengue grave $(9,10)$. La hipoalbuminemia es un indicador de extravasación plasmática. Este fenómeno se genera durante la infección, puesto que los monocitos infectados liberan mediadores vasoactivos que aumentan la permeabilidad vascular, lo que disminuye los niveles de proteínas en sangre (1).

Aunque los niveles de la CK en anteriores estudios mostraron asociación con la gravedad de la enfermedad $(9,10)$, en este estudio no se encontraron diferencias entre los grupos evaluados. Es posible que esto sucediera en los pacientes evaluados porque presentaban alguna enfermedad de base que no fue considerada inicialmente como criterio de exclusión y que que pudo haber alterado los niveles de la enzima, enmascarando el aumento de la síntesis que posiblemente ocurre durante la infección.

El serotipo viral y el tipo de infección (primaria o secundaria), son variables que podrían afectar los resultados del estudio, pues estas han mostrado una fuerte asociación con la gravedad de la enfermedad en estudios anteriores (22-24).

Finalmente, los hallazgos de este estudio sugieren una asociación de los niveles de PCR, LDH y albúmina con la gravedad del dengue. Estas pruebas bioquímicas son de fácil acceso y podrían utilizarse como herramientas predictoras del curso clínico de la infección, con el objetivo de ofrecer una atención oportuna y específica a los pacientes con dengue que consultan tempranamente. Sin embargo, estos resultados deben ser nuevamente evaluados en estudios de cohorte similares conducidos en otras regiones endémicas de dengue.

\section{Agradecimientos}

A Beatriz Parra, por su valioso trabajo en el proceso de diagnóstico de los pacientes; a Janeth Flórez, Diana Marín y Lucelly López, por el apoyo en el manejo de los datos y análisis de la información; igualmente, a la bacterióloga Luz Aida Rey, por su colaboración en la realización de las pruebas bioquímicas.

\section{Conflicto de interés}

Los autores declaramos no tener conflictos de interés.

\section{Financiación}

Este estudio está anidado en la cohorte de pacientes del proyecto $\mathrm{N}^{\circ} 102-459-21561$, financiado por el Departamento Administrativo de Ciencia, Tecnología e Innovación, Colciencias, convenio N¹02-459-21561.

\section{Grupos de investigación participantes en la Red AEDES}

Grupo de Epidemiologia Clínica de La Universidad Industrial de Santander, CINTROP, Centro de Investigación en Enfermedades Parasitarias, Tropicales e Infecciosas y Observatorio Epidemiológico de Enfermedades Cardiovasculares de la Universidad Industrial de Santander, CIDEIM, Centro Internacional de Entrenamiento e Investigaciones, Asociación de Centros de Investigación y Desarrollo Tecnológico, Grupo de Enfermedades Tropicales de la Universidad del Norte, Grupo de Inmunovirología de la Universidad de Antioquia, Grupo Epidemiología y Salud Poblacional y Grupo VIREM Virus Emergentes y Enfermedad de la Universidad del Valle, Grupo de Parasitología y Medicina Tropical de la Universidad Surcolombiana, Centro de Investigación y Diagnóstico de Enfermedades Infecciosas CDI, Grupo GIEPATI Investigación en Enfermedades Parasitarias Tropicales e Infecciosas de la Universidad de Pamplona, University of Michigan, Massachusetts Institute of Technology y Johns Hopkins University.

\section{Referencias}

1. Gubler D. Dengue and dengue hemorrhagic fever. Clin Microbiol Rev. 1998;11:480-96.

2. World Health Organization. Dengue and severe dengue. Fecha de Consulta: 12 de marzo de 2012. Disponible en: http://www.who.int/mediacentre/factsheets/fs117/en/

3. World Health Organization. Dengue guidelines for diagnosis, treatment, prevention and control. Third edition. Geneva: WHO; 2009. p. 3-47.

4. Organización Panamericana de la Salud. Dengue y dengue hemorrágico en las Américas: guías para su prevención y control. Washington, D.C.: OPS; 1995. p. 1-10.

5. Morrow DA, De Lemos JA. Benchmarks for the assessment of novel cardiovascular biomarkers. 
Circulation.2007;115:949-52.http://dx.doi.org/10.1161/ CIRCULATIONAHA.106.683110

6. Itoda I, Masuda G, Suganuma A, Imamura A, Ajisawa A, Yamada K, et al. Clinical features of 62 imported cases of dengue fever in Japan. Am J Trop Med Hyg. 2006;75:470-4.

7. Ahmed MM. Clinical profile of dengue fever infection in King Abdul Aziz University Hospital Saudi Arabia. J Infect Dev Ctries. 2010;4:503-10. http://dx.doi.org/10.3855/jidc.1038

8. Krippner R, Hanish G, Kretschmer H. Dengue fever with hemorrhagic manifestations after a stay in Thailand. Dtsch Med Wochenschr. 1990;115:858-62. http://dx.doi. org/10.1055/s-2008-1065091

9. Villar-Centeno LA, Díaz-Quijano FA, Martínez-Vega RA Biochemical alterations as markers of dengue hemorrhagic fever. Am J Trop Med Hyg. 2008;78:370-4.

10. Ray G, Kumar V, Kapoor AK, Dutta AK, Batra S. Status of antioxidants and other biochemical abnormalities in children with dengue fever. J Trop Pediatr. 1999;45:4-7. http://dx.doi. org/10.1093/tropej/45.1.4

11. Malheiros SM, Oliveira AS, Schmidt B, Lima JG, Gabbai AA. Dengue: Muscle biopsy findings in 15 patients. Arq Neuro-Psiquiatr. 1993;5:159-64. http://dx.doi.org/10.1590/ S0004-282X1993000200001

12. Misra UK, Kalita J, Maurya PK, Kumar P, Shankar SK, Mahadevan A. Dengue-associated transient muscle dysfunction: Clinical, electromyography and histopathological changes. Infection. 2012;40:125-30. http:// dx.doi.org/10.1007/s15010-011-0203-8

13. Levy A, Valero N, Espina LM, Añez G, Arias J, Mosquera J. Increment of interleukin 6 , tumour necrosis factor alpha, nitric oxide, C-reactive protein and apoptosis in dengue. Trans R Soc Trop Med Hyg. 2010;104:16-23. http://dx.doi. org/10.1016/j.trstmh.2009.06.013,

14. Seet RC, Lee CY, Lim EC, Quek AM, Yeo LL, Huang $\mathrm{SH}$, et al. Oxidative damage in dengue fever. Free Radic Biol Med. 2009;47:375-80.http://dx.doi.org/10.1016/j.freerad biomed. 2009.04.035

15. Juffrie M, Meer GM, Hack CE, Haasnoot K, Sutaryo, Veerman AJ, et al. Inflammatory mediators in dengue virus infection in children: Interleukin-6 and its relation to $\mathrm{C}$-reactive protein and secretory phospholipase A2. Am J Trop Med Hyg. 2000;65:70-5.
16. Chaturvedi UC, Agarwal R, Elbishbishi EA, Mustafa AS. Cytokine cascade in dengue hemorrhagic fever: Implications for pathogenesis. FEMS Immunol Med Microbiol. 2000;28:183-8. http://dx.doi.org/10.1111/j.1574695X.2000.tb01474.x

17. Olaison L, Hogevik H, Alestig K. Fever, C-reactive protein, and other acute-phase reactants during treatment of infective endocarditis. Arch Intern Med. 1997:157:885-92. http://dx.doi.org/10.1001/archinte.1997.00440290069007.

18. Roine I, Ledermann W, Arrizaga N, Bosch P, Bertin L, Urrutia S, et al. C-reactive protein in Measles. J Trop Pediatr. 1992;38:149-52. http://dx.doi.org/10.1007/s00431004-1442-9

19. Agrawal DK, Tandon P, Chaturvedi UC, Kumar A. Biochemical study of certain enzymes and metabolites of the carbohydrate metabolism in the skeletal muscle of the dengue virus-infected mice. J Gen Virol. 1978;40:399-408. http://dx.doi.org/10.1099/0022-1317-40-2-399

20. Kalayanarooj S, Vaughn DW, Nimmannitya S, Green S, Suntaya-korn S, Kunentrasai N, et al. Early clinical and laboratory indicators of acute dengue illness. J Infect Dis. 1997;176:313-32. http://dx.doi.org/10.1086/514047

21. Kuo CH, Tai DI, Chang-Chien CS, Lan CK, Chiou SS, Liaw YF. Liver biochemical tests and dengue fever. Am J Trop Med Hyg. 1992;47:265-70.

22. Fried JR, Gibbons RV, Kalayanarooj S, Thomas SJ, Srikiatkhachorn A, Yoon IK, et al. Serotype-specific differences in the risk of dengue hemorrhagic fever: An analysis of data collected in Bangkok, Thailand from 1994 to 2006. PLoS Negl Trop Dis. 2010;4:e617. http://dx.doi. org/10.1371/journal.pntd.0000617

23. Endy TP, Nisalak A, Chunsuttitwat $S$, Vaughn DW, Green $\mathrm{S}$, Ennis FA, et al. Relationship of preexisting dengue virus (DV) neutralizing antibody levels to viremia and severity of disease in a prospective cohort study of DV infection in Thailand. J Infect Dis. 2004;189:990-1000. http://dx.doi. org/10.1086/382280

24. Libraty DH, Acosta LP, Tallo V, Segubre-Mercado E, Bautista A, Potts JA, et al. A prospective nested casecontrol study of Dengue in infants: Rethinking and refining the antibody-dependent enhancement dengue hemorrhagic fever model. PLoS Med. 2009;6:e1000171. http://dx.doi. org/10.1371/journal.pmed.1000171 